\title{
Diagnosis, treatment and survival of uterine sarcoma: A retrospective cohort study of 122 cases
}

\author{
TATIANE BURLE DE SIQUEIRA FERRONI PLENTZ, ELAINE CRISTINA CANDIDO, LAIS FLAUSINO DIAS, \\ MARIA CAROLINA SZYMANSKI TOLEDO, DIAMA BHADRA VALE and JULIO CESAR TEIXEIRA
}

Department of Obstetrics and Gynecology, University of Campinas (UNICAMP), Campinas, 13083-881 São Paulo, Brazil

Received January 24, 2020; Accepted August 11, 2020

DOI: $10.3892 / \mathrm{mco} .2020 .2151$

\begin{abstract}
The present study aimed to assess the diagnosis, treatment and follow-up of uterine sarcoma cases. A retrospective cohort study with 122 women recruited between 2001 and 2016 was performed. The data regarding epidemiology, clinical presentation, treatment and follow-up were analyzed based on the following histological types: Carcinosarcoma, leiomyosarcoma, endometrial stromal sarcoma (ESS) and adenosarcoma. Statistical analysis included descriptive statistics, logistic regression and survival curves. The diagnosis of uterine sarcoma exhibited an increasing trend of +1.2 new cases every 2 years $(\mathrm{P}=0.044)$ and comprised $10 \%$ of all uterine cancer diagnoses. There were $47 \%$ carcinosarcomas, $22 \%$ leiomyosarcomas, $16 \%$ ESS and $14 \%$ adenosarcomas. The majority of the women was $\geq 60$ years old $(62 \%)$. Among the subjects, $77 \%$ were postmenopausal, $61 \%$ had a body mass index up to $29.9 \mathrm{~kg} / \mathrm{m}^{2}$ and $71 \%$ presented with a comorbidity. Regression analysis exhibited an association between post menopause and the histological type associated with lower overall survival (OS), namely leiomyosarcoma or carcinosarcoma (odds ratio, 5.45, $\mathrm{P}<0.001$ ). Stage I malignancy was present in $44 \%$ and Stage IV in $22 \%$. The treatment included primary surgery in $78 \%$ of the cases, whereas $79 \%$ received adjuvant therapy. Only 55 cases achieved disease control and 20 relapsed (36\%) with a 5-year OS rate of $33 \%$. The OS was lower for carcinosarcoma and leiomyosarcoma (20\%; $\mathrm{P}=0.003$ ). In summary, the present study indicated that the number of uterine sarcoma cases had increased between 2001 and 2016. The majority of the women were $>60$ years old and diagnosed in advanced stages. The postmenopausal status was associated with histological types of poor prognosis. The OS was low and worse for patients with carcinosarcoma and leiomyosarcoma.
\end{abstract}

Correspondence to: Dr Julio Cesar Teixeira, Department of Obstetrics and Gynecology, University of Campinas (UNICAMP), Rua Alexander Fleming 101, Campinas, 13083-881 São Paulo, Brazil E-mail: juliotex@unicamp.br; juliotex10@gmail.com

Key words: sarcoma, uterus, diagnosis, treatment, survival

\section{Introduction}

Uterine sarcomas are a group of rare neoplasms, arising from myometrial or mesenchymal tissue, which represent 3 to $7 \%$ of uterine cancer and tend to affect more elderly women, to exhibit aggressive behavior with fast-growing, early distant metastasis and diagnosed in more advanced stages (1-3). Surgery is considered the basis of the treatment and other treatment modalities, such as chemotherapy or radiation therapy, have shown limited results $(3,4)$.

Sarcomas exhibit diverse histopathology and some cases may have a Mullerian duct origin resulting in heterologous histology. The modifications in their classifications have contributed to a gap of knowledge with regard to risk factors, prognosis, and management $(5,6)$. Based on the latest classification of gynecological malignancies by the World Health Organization in 2014 (7), the main uterine sarcoma types are the following: Leiomyosarcoma, endometrial stromal sarcoma (ESS) and adenosarcoma. Carcinosarcomas, on the other hand, have been recently reclassified as a metaplastic form of endometrial carcinoma, and considered for staging and treatment similarly as a high-grade carcinoma. However, this type of malignancy is still frequently analyzed as a sarcoma in association with the previous types $(7,8)$.

The stage of uterine sarcomas (FIGO-2009) can be considered as the most important prognostic factor and the main strategy for optimal results is to achieve an early diagnosis (9). The need to expand knowledge regarding uterine sarcomas is highlighted by the upward trend in the incidence due to the increased life expectancy (10). The present study aimed to provide comprehensive information regarding the characteristics of the women affected, the tumor pattern, diagnosis and management of various types of uterine sarcomas. The patient survival was also assessed over time based on the histological type.

\section{Patients and methods}

Subjects. This is a cohort study including patients assisted in the Women's Hospital of the University of Campinas (UNICAMP), in Campinas (São Paulo), Brazil from 2001 to 2016. UNICAMP is a teaching and research institution and a referral center for the Brazilian public health system. All surgical procedures were performed or supervised by 
surgeons with vast experience. The pathological evaluation was conducted by pathologists specialized in gynecological malignancies. The cases studied were identified from 1,191 records filtered by the International Classification of Diseases, 10th edition (ICD-10) C.54, regarding uterine corpus malignant neoplasia (11). There were found 123 cases of uterine sarcomas, including carcinosarcomas. Following revision of the medical records, one case was excluded due to the patient undergoing a hysterectomy at another hospital and not returning after initial appointment. Therefore the final sample size was adjusted to 122 cases.

Management definition included clinical and image evaluation and discussion in a multidisciplinary meeting. In case when the surgery was the first approach selected, an extrafascial hysterectomy (Piver type 1, Querleu A) with bilateral adnexectomy was performed and pelvic or retroperitoneal lymphadenectomy was conducted in specific cases. Women with advanced or unresectable disease were managed by chemotherapy and/or radiotherapy according to their clinical performance status. The relevant information on clinical characteristics, neoplasms, treatment, and follow-up was collected.

Statistical analysis. The number of cases assisted overtime was analyzed by the $\chi^{2}$ test. Subsequently, a descriptive analysis using the $\chi^{2}$ test or Fisher exact tests was conducted in the total group according to the histological type of sarcomas (WHO classification) (7). The analyses were performed by age group distribution ( $<50$ years, 50-59 years, $60-69$ years, and 70 years or more), parity $(0 ; 1-2 ; 3-4$, and 5 or more), menopausal status, body mass index in $\mathrm{Kg} / \mathrm{m}^{2}(<25 ; 25-29.9$; 30-34.9; and 35 or more), the presence of comorbidities (diabetes, arterial hypertension, cardiopathy, obstructive pulmonary disease, thyroid disease, or other parameters), smoking (current or past, regardless of the period since quitting), neoplasm stage according to FIGO-2009 or -2014 $(9,12)$, modality of initial treatment (surgery, radiotherapy, chemotherapy, or no treatment) and the results following treatment end (optimal surgery and/or complete clinical response following radiotherapy and/or chemotherapy). Subsequently, ESS and adenosarcoma were considered the specific histological types with optimal prognosis, whereas carcinosarcoma and leiomyosarcoma were the malignancy types with poor outcomes. A logistic regression analysis (polytomous models of proportional risks) was performed based on these considerations. Univariate and multivariate analysis was performed with stepwise selection criteria, calculating odds ratios with $95 \%$ confidence intervals for the association between specific variables and the diagnosis of the less favorable histological type.

The longitudinal analyses considered the overall survival (OS) rate as the period between the diagnosis and the last contact or death, whereas the disease-free survival rate was estimated as the period between the end of treatment and the relapse. Recurrence was confirmed by histopathology, clinical examination or image examination and was classified as pelvic or distant metastasis. Survival curves were constructed by the Kaplan-Meier method and analyzed by the log-rank test. Statistical analysis was performed using the StatsDirect statistical software v3.0 (England, www.statsdirect.com). $\mathrm{P}<0.05$ was considered to indicate a statistically significant difference.
The present study was conducted in compliance with the Brazilian National Health Council and was previously approved by the Research Ethics Committee of the University of Campinas.

\section{Results}

Diagnosis trend of the uterine sarcoma. The number of cases with malignant uterine corpus neoplasms has increased in the period of 2001-2016. Specifically, a trend of +10.8 additional cases was noted every two years for carcinomas $(\mathrm{P}=0.003)$, whereas +1.2 additional cases were reported every two years for sarcomas $(\mathrm{P}=0.044)$. During the period 2001-2002, 90 carcinomas and 10 sarcomas were recorded, while 168 carcinomas and 20 sarcomas were identified during the period 2015-2016. The proportion rate of carcinomas-sarcomas remained relatively stable in the period with average values of approximately 90 and $10 \%$, respectively.

Patients and tumor characteristics. The histological type distribution of the 122 sarcoma cases included $47 \%$ carcinosarcomas, $22 \%$ leiomyosarcomas, $16 \%$ ESSs and $14 \%$ adenosarcomas.

The signs or symptoms associated with the diagnosis of sarcomas are presented in Table I. The classification according to the histological type is also shown. Abnormal uterine bleeding and abnormal image examination were noted in 77 and $7 \%$ of the cases, respectively. The diagnosis was confirmed through uterine curettage in $35 \%$ of the subjects, surgery in $34 \%$, hysteroscopy in $13 \%$ and biopsy at a vaginal examination in $17 \%$. A total of 16 cases of sarcoma were diagnosed following hysterectomy due to myomatosis, and all surgeries were performed in women aged between 29-55 years.

The demographic characteristics of the women according to the histological type of sarcoma are described in Table II. The age of the patients was 60 years or higher for $62 \%$ of the total sample size, of which $46 \%$ of carcinosarcomas were present in patients over 70 years, while $26 \%$ of leiomyosarcomas and $45 \%$ of ESSs were detected at an age lower than 50 years $(\mathrm{P}<0.0001)$. The carcinosarcoma group exhibited 2-3 times higher nulliparous subjects $(P=0.217)$ and $47 \%$ of the ESS cases exhibited premenopausal status $(\mathrm{P}<0.001)$.

Logistic regression analysis demonstrated that leiomyosarcoma or carcinosarcoma types (poor prognosis) were significantly associated with a mean age of 50 years or higher and with menopause status. These types of cancer were also associated with a comorbidity as determined by univariate analysis. Menopause was significantly associated with the sarcoma cases as demonstrated by the multivariate regression analysis (OR 5.45; 95\% CI 2.40-12.38; P<0.001).

Staging and management of the sarcomas. The sarcomas were diagnosed at Stage I and IV in 44 and $22 \%$ of the cases, respectively. The stage distribution by histological type is shown in Table III.

The hysterectomy surgery was the first treatment for 95 (78\%) patients and was performed with retroperitoneal lymphadenectomy in 19 patients (14 cases of carcinosarcoma) and radiotherapy and/or chemotherapy in 75 cases (ranging from 59 to $66 \%$ per histological type). The optimal control of the 
Table I. Distribution of signs or symptoms associated with the initial diagnosis of uterine sarcoma according to the histological type.

Uterine sarcomas by histological type

\begin{tabular}{|c|c|c|c|c|c|c|c|c|c|c|}
\hline \multirow[b]{2}{*}{ Signs or symptoms } & \multicolumn{2}{|c|}{$\operatorname{CCS}(n=57)$} & \multicolumn{2}{|c|}{ LMS (n=27) } & \multicolumn{2}{|c|}{$\operatorname{ESS}(n=20)$} & \multicolumn{2}{|c|}{$\operatorname{ADS}(n=17)$} & \multicolumn{2}{|c|}{ Total $(n=122)$} \\
\hline & $\mathrm{n}$ & $\%$ & $\mathrm{n}$ & $\%$ & $\mathrm{n}$ & $\%$ & $\mathrm{n}$ & $\%$ & $\mathrm{n}$ & $\%$ \\
\hline Increased abdominal girth & 2 & 4 & 4 & 15 & 1 & 5 & 0 & 0 & 7 & 6 \\
\hline Pain & 1 & 2 & 4 & 15 & 0 & 0 & 0 & 0 & 5 & 4 \\
\hline Abnormal uterine bleeding & 49 & 85 & 15 & 55 & 18 & 90 & 12 & 70 & 94 & 77 \\
\hline Vaginal discharge & 2 & 4 & 0 & 0 & 0 & 0 & 0 & 0 & 2 & 2 \\
\hline Prolapsed uterine tumor & 0 & 0 & 0 & 0 & 0 & 0 & 3 & 18 & $4^{\mathrm{a}}$ & 3 \\
\hline Image exam & 3 & 5 & 3 & 11 & 1 & 5 & 2 & 12 & 9 & 7 \\
\hline Metastasis & 0 & 0 & 1 & 4 & 0 & 0 & 0 & 0 & 1 & 1 \\
\hline
\end{tabular}

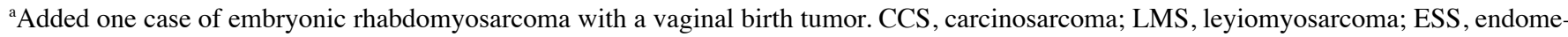
trial stromal sarcoma; ADS, adenosarcoma.

Table II. Distribution of some variables associated with 122 cases of uterine sarcomas managed between 2001 and 2016.

\begin{tabular}{|c|c|c|c|c|c|c|c|c|c|c|c|}
\hline \multirow[b]{3}{*}{ Variables } & \multicolumn{11}{|c|}{ Uterine sarcomas by histological type } \\
\hline & \multicolumn{2}{|c|}{$\operatorname{CCS}(n=57)$} & \multicolumn{2}{|c|}{ LMS $(n=27)$} & \multicolumn{2}{|c|}{$\operatorname{ESS}(n=20)$} & \multicolumn{2}{|c|}{$\operatorname{ADS}(n=17)$} & \multirow[b]{2}{*}{ P-value } & \multicolumn{2}{|c|}{ Total $(n=122)^{b}$} \\
\hline & $\mathrm{n}$ & $\%$ & $\mathrm{n}$ & $\%$ & $\mathrm{n}$ & $\%$ & $\mathrm{n}$ & $\%$ & & $\mathrm{n}$ & $\%$ \\
\hline Age group, years & & & & & & & & & $<0.0001$ & & \\
\hline$<50$ & 0 & 0 & 7 & 26 & 9 & 45 & 3 & 18 & & $20^{\mathrm{b}}$ & 16 \\
\hline $50-59$ & 14 & 24 & 9 & 33 & 1 & 5 & 3 & 18 & & 27 & 22 \\
\hline $60-69$ & 17 & 30 & 7 & 26 & 4 & 20 & 5 & 29 & & 33 & 27 \\
\hline$>70$ & 26 & 46 & 4 & 15 & 6 & 30 & 6 & 35 & & 42 & 35 \\
\hline Parity $^{\mathrm{c}}$ & & & & & & & & & 0.217 & & \\
\hline 0 & 13 & 23 & 2 & 7 & 2 & 10 & 2 & 12 & & 19 & 15 \\
\hline $1-2$ & 15 & 27 & 7 & 26 & 3 & 15 & 5 & 29 & & 30 & 25 \\
\hline $3-4$ & 11 & 20 & 13 & 48 & 8 & 25 & 3 & 18 & & $36^{\mathrm{b}}$ & 30 \\
\hline $5+$ & 17 & 30 & 5 & 19 & 7 & 35 & 7 & 41 & & 36 & 30 \\
\hline Menopause & & & & & & & & & $<0.001$ & & \\
\hline Yes & 56 & 98 & 17 & 63 & 9 & 47 & 12 & 71 & & 94 & 77 \\
\hline No & 1 & 2 & 10 & 37 & 11 & 53 & 5 & 29 & & $28^{\mathrm{b}}$ & 23 \\
\hline BMI, kg/m² & & & & & & & & & 0.640 & & \\
\hline$<25$ & 16 & 28 & 7 & 26 & 4 & 20 & 2 & 12 & & 29 & 24 \\
\hline $25-29.9$ & 19 & 33 & 10 & 37 & 10 & 50 & 6 & 35 & & 45 & 37 \\
\hline $30-34.9$ & 14 & 25 & 7 & 26 & 5 & 25 & 4 & 24 & & 30 & 25 \\
\hline$>35$ & 8 & 14 & 3 & 11 & 1 & 5 & 5 & 29 & & $18^{\mathrm{b}}$ & 14 \\
\hline Comorbidity & & & & & & & & & 0.023 & & \\
\hline Yes & 47 & 83 & 17 & 63 & 10 & 50 & 10 & 59 & & $85^{\mathrm{b}}$ & 71 \\
\hline No & 10 & 17 & 10 & 37 & 10 & 50 & 7 & 41 & & 37 & 29 \\
\hline Smoking & & & & & & & & & 0.365 & & \\
\hline Yes & 6 & 11 & 6 & 22 & 1 & 5 & 2 & 12 & & 15 & 12 \\
\hline No & 51 & 89 & 21 & 78 & 19 & 95 & 15 & 88 & & $107^{\mathrm{b}}$ & 88 \\
\hline
\end{tabular}



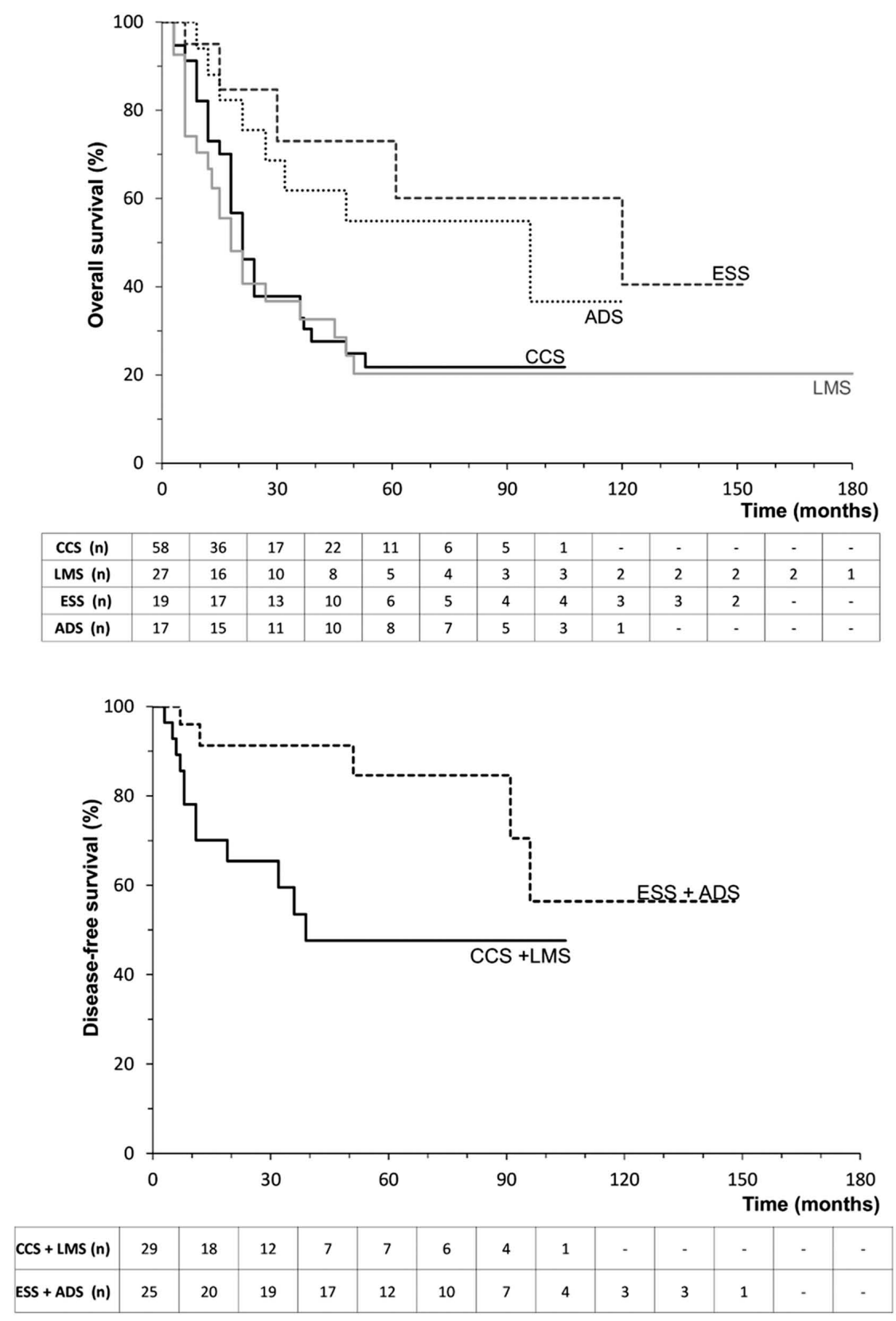

Figure 1. OS ( $\mathrm{n}=121)$ according to histological type. CCS and LMS were associated with poor survival compared with ESS and ADS (log-rank test, P=0.003). Disease-free survival $(n=54)$ according to histological type group. CCS+LMS $(n=29)$ vs. ESS+ADS $(n=25)$, with worse rate for the first group (log-rank test, $\mathrm{P}=0.022$ ). OS, overall survival; CCS, carcinosarcoma; LMS, leiomyosarcoma; ESS, endometrial stromal sarcoma; ADS, adenosarcoma.

disease (complete surgical resection and/or complete clinical response) was achieved in 58\% of the cases (55/95). Following stratification according to the type of sarcoma, this optimal control was increased to $71 \%$ for the adenosarcoma cases, to $67 \%$ for the ESSs, to $37 \%$ for the leiomyosarcoma patients and to $33 \%$ for the carcinosarcoma subjects.

Sarcomas recurrence and survival over time. Relapses occurred in $36 \%(20 / 55)$ of the subjects who reached initial complete disease control. The relapses occurred in the first 12 months in 13 cases and within 36 months in 18 cases. A total of 11 cases were distant metastases. A late relapse was noted after 91 months of follow up, which involved a subcutaneous tissue metastasis (abdominal wall) in a woman with an ESS of low grade, Stage II, and initially managed with surgery and radiotherapy.
A total of 27 women (22\%) were unable to perform surgery upfront. This was predominantly noted in the carcinosarcoma cases (32\%) compared to 12 and $15 \%$ noted for the other types of sarcomas. However, the differences were not significant $(\mathrm{P}=0.108)$. Out of these patients, 21 received initial radiotherapy and/or chemotherapy (without surgery) and there was only one case (2\%) with complete clinical response.

Longitudinal analysis resulted in an OS rate of $76 \%$ in 12 months and 33\% in five years. Further analysis performed according to the histological type demonstrated that the ESSs and adenosarcomas exhibited an OS rate of $88-90 \%$ in 12 months and of 55-61\% in five years. In comparison, carcinosarcomas and leiomyosarcomas presented the worst results with a 5 -year OS of $20-22 \%(\mathrm{P}=0.003$; Fig. 1). The disease-free survival rate was $66 \%$ for all cases with disease control at the 
Table III. Distribution of 122 cases of uterine sarcomas managed between 2001 and 2016 according to the FIGO staging system.

Uterine sarcomas by histological type

\begin{tabular}{|c|c|c|c|c|c|c|c|c|c|c|}
\hline \multirow[b]{2}{*}{ Stage } & \multicolumn{2}{|c|}{$\operatorname{CCS}(n=57)$} & \multicolumn{2}{|c|}{ LMS $(n=27)$} & \multicolumn{2}{|c|}{$\operatorname{ESS}(n=20)$} & \multicolumn{2}{|c|}{$\operatorname{ADS}(n=17)$} & \multicolumn{2}{|c|}{ Total $(\mathrm{n}=122)^{\mathrm{b}}$} \\
\hline & $\mathrm{n}$ & $\%$ & $\mathrm{n}$ & $\%$ & $\mathrm{n}$ & $\%$ & $\mathrm{n}$ & $\%$ & $\mathrm{n}$ & $\%$ \\
\hline \multicolumn{11}{|l|}{ I } \\
\hline Ia & 10 & 18 & 1 & 4 & 6 & 30 & 5 & 29 & 22 & 18 \\
\hline $\mathrm{Ib}$ & 6 & 10 & 10 & 37 & 6 & 30 & $9^{b}$ & 53 & $32^{\mathrm{a}}$ & 26 \\
\hline II & 4 & 7 & 4 & 15 & 4 & 20 & 1 & 6 & 13 & 11 \\
\hline \multicolumn{11}{|l|}{ III } \\
\hline IIIa & 4 & 7 & 0 & 0 & 0 & 0 & 0 & 0 & 4 & 3 \\
\hline $\mathrm{IIIb}$ & 13 & 23 & 1 & 4 & 1 & 5 & 0 & 0 & 15 & 12 \\
\hline IIIc & 6 & 10 & 2 & 7 & 1 & 5 & 1 & 6 & 10 & 8 \\
\hline \multicolumn{11}{|l|}{ IV } \\
\hline IVa & 2 & 4 & 1 & 4 & 0 & 0 & 0 & 0 & 3 & 3 \\
\hline $\mathrm{IVb}$ & 12 & 21 & 8 & 29 & 2 & 10 & 1 & 6 & 23 & 19 \\
\hline
\end{tabular}

${ }^{\mathrm{a} A d d e d} 1$ case in total relative to Stage Ib embryonal rhabdomyosarcoma; ${ }^{\mathrm{b}}$ Adenosarcoma stage Ib: Seven cases with $<50 \%$ invasion and two cases $>50 \%$ of the myometrial thickness. Fisher's exact test for stage I vs. II-IV, P=0.0001. CCS, carcinosarcoma; LMS, leiomyosarcoma; ESS, endometrial stromal sarcoma; ADS, adenosarcoma; FIGO, International Federation of Gynecology and Obstetrics $(9,12)$.

first treatment. This rate was altered from $48 \%$ in five years for the carcinosarcoma plus leiomyosarcoma group to $85 \%$ for the ESS plus adenosarcoma group $(\mathrm{P}=0.022$; Fig. 1$)$.

During the end of the follow-up period, only $25 \%$ (31/122) of the women remained disease-free and survived and $57 \%$ $(69 / 122)$ were deceased due to disease progression. These statistics were higher for leiomyosarcoma (78\%) and carcinosarcoma (61\%) compared with those noted for ESS (30\%) and adenosarcoma $(41 \%)(\mathrm{P}=0.005)$.

\section{Discussion}

Uterine sarcomas have accounted for $10 \%$ of uterine corpus malignancies from 2001-2016. An increasing trend for new cases has been noted for these malignancies, mainly for histological types, such as carcinosarcoma, ESS and adenosarcoma. The increasing number of the cases and the higher proportion of sarcomas observed in comparison to the lower incidence described previously (range 3-7\%) $(3,13)$ may be associated with a higher rate of severe case referrals to reference centers (10).

With regard to diagnosis, $77 \%$ of cases exhibited abnormal uterine bleeding as an 'early' symptom despite the perception that uterine sarcoma is a fast-growing tumor with a pelvic mass. Abnormal uterine bleeding was observed for all histological types and may reflect a delay in diagnosis, resulting in advanced-stage disease and additional bleeding.

The main prevalent histological types were those with a poor prognosis, such as carcinosarcomas (47\%) and leiomyosarcomas (22\%) compared to ESSs (16\%) and adenosarcomas $(14 \%)$. In general, uterine sarcomas predominated in patients over 60 years of age, explaining the high proportion of postmenopausal women and of those with comorbidities. It is noteworthy that $46 \%$ of carcinosarcomas occurred in women older than 70 years, whereas no case under 50 years of age was noted. $26 \%$ of leiomyosarcomas and $45 \%$ of ESSs occurred before 50 years of age $(\mathrm{P}<0.0001)$. These findings are in accordance with recent studies indicating that ESS predominates in the age group of 40-50 years (14-16).

Multivariate regression analysis demonstrated 5-fold increased risk for the incidence of carcinosarcomas and leiomyosarcomas in subjects that were on menopause. These results confirm the association between increased age and risk of sarcomas. However, it remains to be established whether menopause is a risk factor for sarcomas $(17,18)$, despite the evidence suggesting that hormonal changes may cause modifications in mitotic activity and uterine cell morphology (19).

Despite a lack of significant association, all histological types exhibited a high rate of multiparity ( $>50 \%$ with three or more deliveries). In contrast to this finding, $23 \%$ of nulliparity was noted for carcinosarcomas and a history of smoking was present in $22 \%$ of patients with leiomyosarcoma that occurred in less than $12 \%$ of other tumor types. Currently, insufficient evidence is present regarding the association of smoking with sarcoma and conflicting data have been reported. A German study of 143 women demonstrated that $12 \%$ of carcinosarcomas were from patients who were smokers, whereas this percentage was increased to $16 \%$ in leiomyosarcomas and to $28 \%$ in ESSs. A lower OS was noted in smokers (20).

The main prognostic factors for uterine sarcomas are stage and adequate initial approach by surgery $(5,14,21)$. A relatively low proportion was noted for stage I sarcomas (44\%), which was similar to the results reported by a Norwegian study of 1,042 cases (22). Furthermore, Stage I was the predominant stage noted in ESSs (60\%) and adenosarcomas (82\%), while 
$33 \%$ of leiomyosarcomas and $25 \%$ of carcinosarcomas were classified as Stage IV, probably related to histological type aggressiveness $(\mathrm{P}=0.0001)$. Similar findings were noted for leiomyosarcomas in an American study with 219 patients diagnosed with the disease from 1982 to $2005.35 \%$ of these cases were classified as Stage IV tumors (23).

Surgery is considered a standard therapeutic approach for sarcomas and a hysterectomy was performed for $78 \%$ of the patients in the present study. It is important to note that $62 \%$ of these cases also received adjuvant therapy, which was not associated with the histological type. However, it has been reported that additional therapeutic interventions may yield controversial results $(20,24)$. In the present study, only 1 out of 21 patients treated primarily with radiotherapy and/or chemotherapy without surgery, exhibited a satisfactory clinical response, highlighting the poor response of these cases to the standard radiotherapy treatment, which has been previously shown $(14,21,25)$. An additional factor, which was indicative of the aggressiveness of sarcomas and possibly of the delay for diagnosis and management, was the lack of treatment noted in the six untreated cases, which was due to the advanced stage of the disease. A total of 5 out of these 6 cases were carcinosarcomas.

The low OS rate observed was reduced from $76 \%$ in 12 months to $33 \%$ in five years confirming the poor prognosis of uterine sarcomas. The formation of two different groups according to prognosis was evident. This was defined by the different histological types as follows: The first group included the ESS and adenosarcoma and exhibited a high 5-year OS rate $(55-61 \%)$, whereas the second group was composed of carcinosarcoma and leiomyosarcoma cases, which were more aggressive and led to poor outcomes and reduced 5-year OS rate $(20 \%, \mathrm{P}=0.003)$.

Similar findings were reported by several studies with regard to the 5-year OS rate and the histological subtype. A percentage range of $40-46$ and $30-45 \%$ has been reported for leiomyosarcomas carcinosarcomas, respectively. In contrast to these findings, the range and percentage was estimated to $86-97 \%$ for ESSs and to $69 \%$ for adenosarcomas, respectively $(20,26-28)$. The rates reported in the present study were lower than those previously published. These low survival rates were noted for all histological types and may reflect the limited treatment modalities available, the rapid progression of the disease and the delay to the diagnosis and treatment. The latter is a common disadvantage noted in the Brazilian public health system. The increase of the time required for management of an aggressive sarcoma may reduce the low OS rate of the patients.

Among 55 women that achieved remission, 20 women exhibited recurrences (36\%), of which 18 cases recurred in 36 months. Distant metastases were noted in 11 cases. The main sites of metastasis were the lungs ( $n=4$ cases) and the bones ( $n=3$ cases). These findings were in agreement with those noted in previous reports $(14,29)$. Disease-free survival followed the OS pattern for two distinct histological types, with the ESS and adenosarcoma group exhibiting a 5-year survival rate of $85 \%$, against $48 \%$ for the second group (carcinosarcoma and leiomyosarcoma, $\mathrm{P}=0.022$ ).

The limitations of this study include the small number of cases and its retrospective nature. A certain levels of bias existed regarding patient selection from a cancer center and the temporal alteration of the population access could explain the higher prevalence of tumors with a poor prognosis. In contrast to these findings, the advantage of the present study was the appropriate selection of the institution in which the subjects were treated, which included a predefined protocol for staging, treatment, and follow-up, with accurate information recorded, and a minimal loss of follow-up observed (one patient).

In conclusion, the present study demonstrated that the rate of treated uterine sarcoma cases had increased from 2001 to 2016, mainly due to the incidence of different histological types, such as carcinosarcoma, ESS and adenosarcoma, and were predominant in women over 60 years of age and diagnosed in advanced stages. Postmenopause was associated with histological types of poorer prognosis, such as carcinosarcoma and leiomyosarcoma. A low 5-year OS rate was noted and estimated to $33 \%$, which was lower than expected. The survival rate was worse low for carcinosarcoma and leiomyosarcoma. Recurrences occurred in one-third of cases, usually in the first three years following diagnosis. Approximately 50\% of these recurrences were distant metastases. The improvement of the women public health care system and a faster investigation of symptomatic postmenopausal cases are crucial in order to achieve early diagnosis and reduce the unfavorable outcomes observed for uterine sarcomas.

\section{Acknowledgements}

Not applicable.

\section{Funding}

No funding was received.

\section{Availability of data and materials}

The datasets used and/or analyzed during the present study are available from the corresponding author on reasonable request.

\section{Authors' contributions}

TBDSFP contributed to the conception and design of this study, acquisition of data, interpretation of data, and drafting the manuscript. ECC contributed to the conception and design of this study, data revision, and drafting the manuscript. LFD contributed to the acquisition of data and data revision. MCST contributed to the conception and design of this study and interpretation of data. DBV contributed to the methodology development and interpretation of data. JCT contributed to the conception and design of this study, acquisition of data, analysis and interpretation of data, drafting the manuscript, and general supervision. All authors read and approved the final manuscript.

\section{Ethics approval and consent to participate}

The present study was developed in compliance with Resolution 466/12 of the Brazilian National Health Council and Declaration of Helsinki and was previously approved by the Research Ethics Committee of Unicamp (approval no. 1.760 .085 , October 4,2016 ), which waived the requirement for informed consent. 


\section{Patient consent for publication}

Not applicable.

\section{Competing interests}

The authors declare that they have no competing interests.

\section{References}

1. Bray F, Ferlay J, Soerjomataram I, Siegel RL, Torre LA and Jemal A: Global cancer statistics 2018: GLOBOCAN estimates of incidence and mortality worldwide for 36 cancers in 185 countries. CA Cancer J Clin 68: 394-424, 2018.

2. Singh $\mathrm{N}$ and Gilks CB: The changing landscape of gynaecological cancer diagnosis: Implications for histopathological practice in the 21st century. Histopathology 70: 56-69, 2017.

3. Tropé CG, Abeler VM and Kristensen GB: Diagnosis and treatment of sarcoma of the uterus. A review. Acta Oncol 51: 694-705, 2012

4. Ghirardi V, Bizzarri N, Guida F, Vascone C, Constantini B, Scambia G and Fagotti A: Role of surgery in gynaecological sarcomas. Oncotarget 10: 2561-2575, 2019.

5. Giuntoli RL 2nd, Metzinger DS, DiMarco CS, Cha SS, Sloan JA, Keeney GL and Gostout BS: Retrospective review of 208 patients with leiomyosarcoma of the uterus: Prognostic indicators, surgical management, and adjuvant therapy. Gynecol Oncol 89: 460-469, 2003 .

6. Hoang L, Chiang S and Lee CH: Endometrial stromal sarcomas and related neoplasms: New developments and diagnostic considerations. Pathology 50: 162-177, 2018.

7. Kurman RJ, Carcangiu ML, Herrington CS and Young RH (eds) WHO Classification of Tumours of Female Reproductive Organs 4th edition. IARC Press, Lyon, pp135-147, 2014.

8. Oliva E: Cellular mesenchymal tumors of the uterus: A review emphasizing recent observations. Int J Gynecol Pathol 33: 374-384, 2014

9. Prat J: FIGO staging for uterine sarcomas. Int J Gynaecol Obstet 104: 177-178, 2009.

10. Ueda SM, Kapp DS, Cheung MK, Shin JY, Osann K, Husain A Teng NN, Berek JS and Chan JK: Trends in demographic and clinical characteristics in women diagnosed with corpus cancer and their potential impact on the increasing number of deaths. Am J Obstet Gynecol 198: 218, 2008.

11. World Health Organization: International Statistical Classification of Diseases and Related Health Problems 10th Revision. ICD-10 version 2015. Available at: https://icd.who.int/ browse10/2015/en.

12. FIGO Committee on Gynecologic Oncology: FIGO staging for carcinoma of the vulva, cervix, and corpus uteri. Int J Gynecol Obstet 125: 97-98, 2014

13. Koivisto-Korander R, Martinsen JI, Weiderpass E, Lemnen A and Pukkala E: Incidence of uterine leiomyosarcoma and endometrial stromal sarcoma in nordic countries: Results from NORDCAN and NOCCA databases. Maturitas 72: 56-60, 2012.

14. Mbatani N, Olawaiye EA and Prat J: Uterine sarcomas. Int J Gynecol Obstet 143: 51-58, 2018.
15. Felix AS, Cook LS, Gaudet MM, Rohan TE, Schouten LJ, Setiawan VW, Wise LA, Anderson KE, Bernstein L, De Vivo I, et al: The etiology of uterine sarcomas: A pooled analysis of the epidemiology of endometrial cancer consortium. Br J Cancer 108: 727-734, 2013.

16. Zhang YY, Li Y, Qin M, Cai Y, Jin Y and Pan LY: High-grade endometrial stromal sarcoma: A retrospective study of factors influencing prognosis. Cancer Manag Res 11: 831-837, 2019.

17. Ishidera Y, Yoshida H, Oi Y, Katayama K, Miyagi E, Hayashi H and Shigeta $\mathrm{H}$ : Analysis of uterine corporeal mesenchymal tumors occurring after menopause. BMC Women Health 19: 13, 2019.

18. Koivisto-Korander R, Butzow R, Koivisto AM and Leminen A: Clinical outcome and prognostic factors in 100 cases of uterine sarcoma: Experience in helsinki university central hospital 1990-2001. Gynecol Oncol 111: 74-81, 2008.

19. Oliva E: Practical issues in uterine pathology from banal to bewildering: The remarkable spectrum of smooth muscle neoplasia. Mod Pathol 29 (Suppl 1): S104-S120, 2016.

20. Burghaus S, Halmen S, Gass P, Mehlhorn G, Schrauder MG, Lux MP, Renner SP, Beckmann MW, Hein A and Thiel FC: Outcome and prognosis in uterine sarcoma and malignant mixed mullerian tumor. Arch Gynecol Obstet 294: 343-351, 2016.

21. Menczer J: Review of recommended treatment of uterine carcinoma. Curr Treat Options Oncol 16: 53, 2015.

22. Nordal RR and Thoresen SO: Uterine sarcomas in Norway 1956-1992: Incidence, survival and mortality. Eur J Cancer 33: 907-911, 1997.

23. Zivanovic O, Leitao MM, Iasonos A, Jacks LM, Zhou Q, Abu-Rustum NR, Soslow RA, Juretzka MM, Chi DS, Barakat RR, et al: Stage-specific outcomes of patients with uterine leiomyosarcoma: A comparison of the international federation of gynecology and obstetrics and american joint committee on cancer staging systems. J Clin Oncol 27: 2066-2072, 2009.

24. Wada H, Enomoto T, Fujita M, Yoshino K, Nakashima R, Kurachi H, Haba T, Wakasa K, Shroyer KR, Tsujimoto M, et al: Molecular evidence that most but not all carcinosarcomas of the uterus are combination tumors. Cancer Res 57: 5379-5385, 1997.

25. Nusrath S, Bafna S, Rajagopalan R, Thammineedi SR, Raju KVV, Patnaik SC, Pawar S, Reddy Y, Chavali RN and Murthy SS: Uterine sarcomas: Experience from a tertiary cancer care center from India. Indian J Surg Oncol 10: 342-349, 2019.

26. Şükür YE, Taşkın S, Varlı B, Ateş C, Güngör M and Ortaç F: Prognostic factors for disease-free and overall survival of patients with uterine carcinosarcoma. Int J Clin Oncol 23: 114-120, 2018.

27. Bernard B, Clarke BA, Malowany JI, McAlpine J, Lee CH, Atenafu EG, Ferguson S and Mackay H: Uterine adenosarcomas: A dual-institution update on staging, prognosis and survival. Gynecol Oncol 131: 634-639, 2013.

28. Pietzner K, Buttmann-Schweiger N, Sehouli J and Kray winkel K: Incidence patterns and survival of gynecological sarcoma in Germany: Analysis of population-based cancer registry data on 1066 women. Int J Gynecol Cancer 28: 134-138, 2018.

29. D'Angelo E and Prat J: Uterine sarcomas: A review. Gynecol Oncol 116: 131-139, 2010. 